\title{
A brief introduction to the COM-B Model of behaviour and the PRIME Theory of motivation
}

Robert West ${ }^{1}$, Susan Michie

1 Univers ity College Lond on, University of London

\begin{abstract}
The COM-B model of behaviour is widely used to identify what needs to change in order for a behaviour change intervention to be effective. It identifies three factors that need to be present for any behaviour to occur: capability, opportunity and motivation. These factors interact over time so that behaviour can be seen as part of a dynamic system with positive and negative feedback loops. Motivation is a core part of the model and the PRIME Theory of motivation provides a framework for understanding how reflective thought processes (Planning and Evaluation processes) and emotional and habitual processes (Motive and Impulse/inhibition processes) interact at every moment leading to behaviour (Responses) at that moment.
\end{abstract}

Changing human behaviour is crucial to meeting the challenges facing society, including the climate emergency, pandemics, violence, and addictive behaviours such as tobacco use. A model of behaviour has been developed that aims to assist developers of behaviour change interventions to identify appropriate targets for those interventions: the COM-B model. Linked to this model is a broad model of motivation that aims to describe how reflective thought processes and emotional and habitual factors interact in each moment to drive motivation to act in particular ways. This paper provides a brief summary of the COM-B and PRIME models. Fuller details can be found in .

The COM-B Model of behaviour

The COM-B model represents the observation that at any given moment, a particular behaviour will occur only when the person concerned has the capability and opportunity to eng age in the behaviour and is more motivated to enact that behaviour than any other behaviours $^{[1]}$. Figure 1 illustrates the model including definitions of the entities involved and specification of the relationships between them. 
Fourthly, motivation can be thought of as a quantity attaching to any behaviours that capability and opportunity would permit in a given situation. In theory there could be millions of these but in practice in any given situation there are only a small number that the brain will be in some sense considering, either though habit or desire. These behaviours may or may not enter conscious awareness and indeed most of the time our actions seem to us simply to flow from stimuli in our environment and our thoughts. But motivation always involves a competition between alternative behaviours which means that sometimes getting someone to enact a behaviour can involve decreasing motivation to enact a competing behaviour as much as increasing motivation to enact the behaviour itself.

\section{The PRIME Theory of motivation}

The PRIME Theory of motivation was developed to bring into a single framework, the large number of models and theories of motivation currently being used, including ones that focus on reflective choice processes, ones that focus on emotions and drives, and ones that focus on habit and instinct ${ }^{[2]}$. It is not a replacement for these more specific theories, but a way in which they can be brought together to help address the diversity of factors that influence motivation and therefore behaviour.

At the heart of the theory is a representation of how the 'human motivational system' is structured. Figure 2 shows this in schematic form.

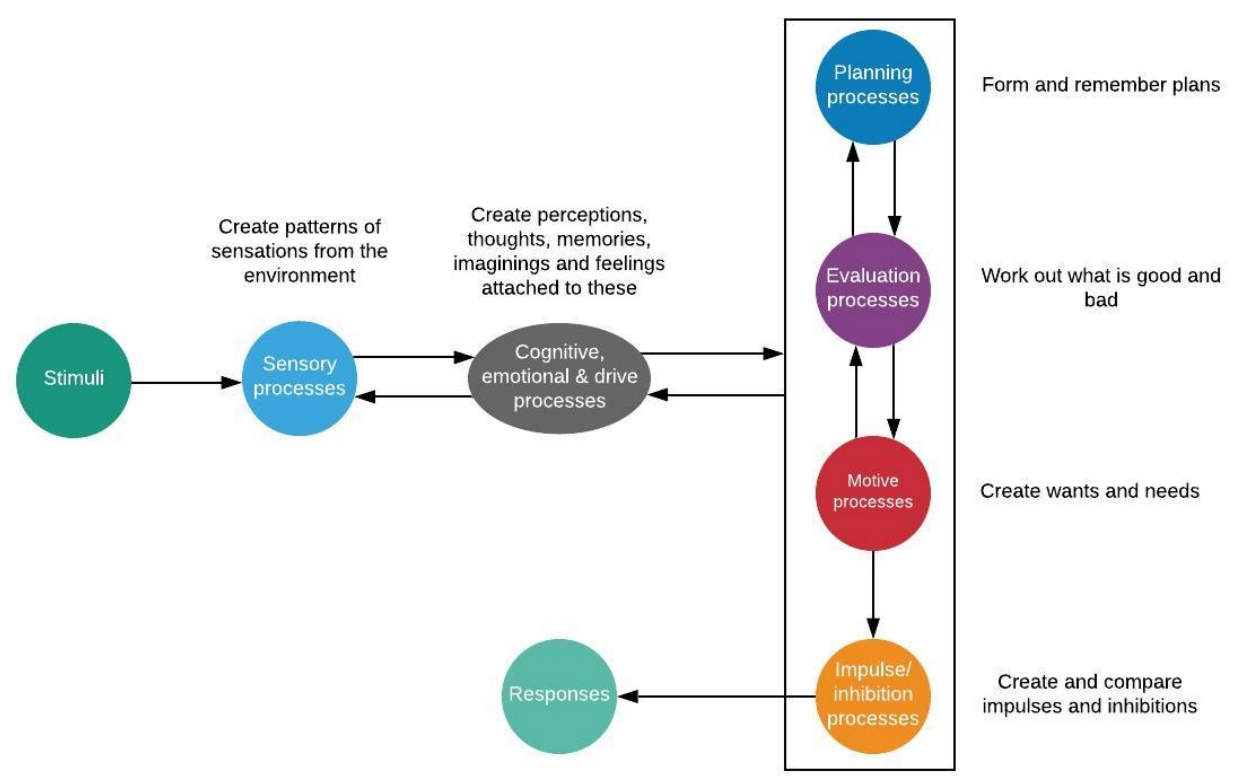

Figure 2: The structure of human motivation according to PRIME Theory 
Behaviours consists of responses that are generated by brain processes that create and compare potentially competing or reinforcing impulses and inhibitions.

Impulses are patterns of brain activity that coordinate and impel responses while inhibitions are patterns of brain activity that work directly oppose these.

Impulses and inhibitions are generated, either directly from input from sensory or perceptual processes though learned (habit) or innate (instinct) associations, or they are generated by motives.

Motives are feelings of 'want' or 'need'. Wants are feeling of attraction arising from anticipated pleasure or satisfaction; needs are feelings of attraction generated by anticipated relief from mental or physical discomfort. Thus wants and needs lie at the heart of all goal directed human behaviour. As such PRIME theory recognises a fundamental principle of human behaviour which that at every moment we act in pursuit of what we most want or need at that moment'. When attempting to change behaviour by chang ing motivation, a key target is the momentary wants and needs that will be experienced at the moment when the behaviour becomes appropriate.

Wants and needs are generated by processes involving associations with positive and neg ative emotions and by drive states such as hunger and thirst. They are also driven to some extent by evaluations.

Evaluations are beliefs that involve some form of judgement about 'goodness' or 'badness'. Thus they include beliefs about what is right and wrong, good or bad, beneficial or harmful. It is important to distinguish between beliefs as processes that are active at a given moment and 'belief dispositions' that involve a propensity to generate such beliefs under certain conditions. This is because unless a belief is active at a given moment it will not directly influence motives and therefore not directly influence behaviour.

Evaluations are generated by sensory processes and perception, memory, and by inference of some form (not necessarily logical). They are also influenced by wants and needs - we tend to believe what we want or need to believe. Important evaluations are beliefs about what we 'should' and 'should not' do: what may be termed 'imperatives'. These are beliefs about behaviours that are judged to be good or bad. However, these only influence behaviour if they lead to corresponding motives - feelings of wanting or 
needing to do the behaviour at a particular moment.

There are certain kinds of evaluation that apply to a wide range of situations and we term these 'values'. Values can relate to classes of behaviour (e.g. lying is bad), objects (e.g. human life is important), or abstract concepts (e.g. justice is good). These play an important role in behaviour, but only if they translate into specific evaluations and imperatives relevant to the situation at the relevant moment. If they conflict with another evaluation or a motive they can either be overridden or an 'exception' will be created which allows the desired action to occur despite the value (e.g. lying is bad but in this case it would be a kindness to praise a child for a drawing that has no artistic value to avoid the child being upset and the parent being angry).

Aside from imperatives being generated by emotional and thoug ht processes described above, they are also generated by remembering 'plans'. Plans are self-conscious intentions to enact behaviours under certain conditions, e.g. at a given point in time or in response to a given set of circumstances. Plans are generated when an imperative is formed but the appropriate conditions for the behaviour are not present at the current moment in time. The imperative is stored in memory, or some memory aid, with the aim of being activated at the appropriate moment.

Plans form the overarching structure of human behaviour and are represented in diaries and physical or mental to-do lists. There are some kinds of plans that relate to circumstances or events that repeat over time. These can be termed 'personal rules'. Personal rules represent the practical and moral precepts governing our behaviour and as such are extremely important influences on it. These include such diverse behaviours as always brushing one's teeth for 2 minutes on getting up in the morning and before going to bed, and not eating red meat.

Like beliefs, it is important to distinguish plans as active processes at a given moment and plan dispositions, that are in a sense stored plans, ready to be activated at the appropriate time. Thus plans, and their subclass personal rules, can only influence behaviour if they are activated at the relevant moment. They also have to generate imperatives ( $a$ belief that the action should be undertaken) at that moment, which then has to generate a want or need to enact the behaviour and ultimate the impulse to engage in it. Therefore, plan dispositions (that can be thought of as stored plans) can only influence behaviour if they are remembered when required, people still think the behaviour is worth doing and still wants of need to do it. At each point in this process it 
could be coming into competition with other plans, imperatives and wants and needs. This is an important reason why so many of our intentions fail to be enacted.

The paragraphs above describe the structure of the human motivational system. PRIME Theory recognises a number of important principles of its operation, and the kinds of things that generate wants and needs. Two of these are considered to be particularly worthy of note. One is identity and the other is modelling or imitation.

Identity consists of all thoughts and images we have pertaining to ourselves are we were, are or could be, together with feelings attaching to these. It is particularly important as a source of wants or needs. This is because a sense of self is essential for the generation of plans, but also because we are highly salient and usually highly valued objects in our world. What happens to us and who we consider ourselves to be creates strong positive and negative affect.

Modelling and imitation are important because humans are highly imitative and evidence indicates that we mimic others, even without conscious awareness. This modelling or imitation appears to operate at all levels of behaviour and thought, from mannerisms and accents to values and personal rules.

\section{Conclusions}

The COM-B model of behaviour and the PRIME Theory of motivation provide a basis for developing behaviour change interventions that recognise the importance of all the relevant factors influencing behaviour. They are general models and provide guidance on broad strategies. COM-B and PRIME need to be used in conjunction with more specific models relevant to the specific behaviours in question.

\section{References}

1. ^ Susan Michie, Maartje M van Stralen, Robert West. (2011). The behaviour change wheel: A new method for characterising and designing behaviour change interventions. Implementation Sci, vol. 6 (1). doi:10.1186/1748-5908-6-42.

2. ^ Robert West, Jamie Brown. (2013). Theory of Addiction. John Wiley \& Sons. 